\title{
Stability of Nonlinear Stochastic Discrete-Time Systems
}

\author{
Yan Li, ${ }^{1}$ Weihai Zhang, ${ }^{2}$ and Xikui Liu' ${ }^{1}$ \\ ${ }^{1}$ College of Information Science \& Engineering, Shandong University of Science and Technology, Qingdao, Shandong 266590, China \\ ${ }^{2}$ College of Information and Electrical Engineering, Shandong University of Science and Technology, Qingdao, Shandong 266590, China
}

Correspondence should be addressed to Weihai Zhang; w_hzhang@163.com

Received 6 May 2013; Accepted 12 July 2013

Academic Editor: Baocang Ding

Copyright (c) 2013 Yan Li et al. This is an open access article distributed under the Creative Commons Attribution License, which permits unrestricted use, distribution, and reproduction in any medium, provided the original work is properly cited.

This paper studies the stability for nonlinear stochastic discrete-time systems. First of all, several definitions on stability are introduced, such as stability, asymptotical stability, and pth moment exponential stability. Moreover, using the method of the Lyapunov functionals, some efficient criteria for stochastic stability are obtained. Some examples are presented to illustrate the effectiveness of the proposed theoretical results.

\section{Introduction}

Stability is the first of all the considered problems in the system analysis and synthesis of modern control theory, which plays an essential role in dealing with infinite-horizon linear-quadratic regulator, $\mathrm{H}_{2} / \mathrm{H}_{\infty}$ robust optimal control, and other control problems; see [1-5]. In 1892, Lyapunov introduced the concept of stability of dynamic systems and created a very powerful tool known as the Lyapunov method in the study of stability. It can be found that the Lyapunov method has been developed and applied to investigate stochastic stability of the Itô-type systems, and many important classical results on deterministic differential equations have been generalized to the stochastic Itô systems; we refer the reader to Arnold [6], Friedman [7], Has'minskii [8], Kushner [9], Kolmanovskii and Myshkis [10], Ladde and Lakshmikantham [11], Mohammed [12], and Mao [13].

Compared with the plenty of fruits of the continuoustime Itô systems, few results have been obtained on the stability of discrete-time nonlinear stochastic systems:

$$
x(t+1)=f(x(t), w(t), t) .
$$

In [14], the mean square stability of the discrete-time time-varying Markov jump system

$$
x(t+1)=\left[A_{0}\left(t, \eta_{t}\right)+\sum_{k=1}^{r} A_{k}\left(t, \eta_{t}\right) w_{k}(t)\right] x(t)
$$

was studied. Nevertheless [15], based on the exact observability assumption, extensively researched the mean square stability of the following linear discrete-time time-invariant system with multiplicative noise:

$$
x(t+1)=A x(t)+B x(t) w(t),
$$

where the Classical Lyapunov Theorem was extended. Reference [16] considered the $p$ th mean stability of the following difference equations

$$
\begin{gathered}
x(t+1)=A(t, \omega) x(t), \quad t=0,1,2, \ldots, \\
x(t+1)=A(t, \omega) x(t)+f(t, x(t)), \quad t=0,1,2, \ldots,
\end{gathered}
$$

with random coefficients. For the nonlinear stochastic difference equation

$$
\begin{aligned}
x(t+1)= & \sum_{j=0}^{t+h} a_{j} x(t-j)+\sum_{j=0}^{t+h} \sigma_{j} x(t-j) \xi(t) \\
& +g(t, x(-h), \ldots, x(t)),
\end{aligned}
$$

its stability in probability was investigated in [17]. It is not difficult to find that, different from the continuous-time Itô systems, up to now, there lacks the systematic theory on stability of nonlinear discrete-time stochastic systems. The aim of this paper is to develop a parallel theory for stability of general nonlinear stochastic discrete-time systems, and some sufficient criteria for various stabilities are given. 
Different from the Itô systems, most sufficient criteria are presented via $\mathscr{L} V(x) \leq 0$ or $\mathscr{L} V(x)<0$ together with other assumptions on the Lyapunov function $V(x)$, where $\mathscr{L}$ is the so-called infinitesimal generator associated with the given Itô system. In discrete-time stochastic systems, most stability criteria are given via $E[\Delta V(x(t))] \leq 0$ or $E[\Delta V(x(t))]<0$, where $E$ represents the mathematical expectation. So general discrete stochastic stability is more difficult to be tested due to the appearance of the mathematical expectation $E$.

The organization of this paper is as follows. Section 2 presents some stability definitions. Section 3 is devoted to developing some efficient criteria for various stabilities. Section 4 contains three examples provided to show the efficiency of the proposed results. Finally, we end this paper by Section 5 with a brief conclusion.

For convenience, we adopt the following notations:

$A^{T}$ : the transpose of the matrix $A$;

$A \geq 0(A>0): A$ is a positive semidefinite (positive definite) matrix;

$D_{r}:=\left\{x \in R^{n}:|x|<r\right\}$ for $r>0$;

$C^{2}(U)$ : the class of functions $V(x)$ twice continuously differential with respect to $x \in U$;

$Z^{+}:=\{0,1,2, \ldots\}$;

$P\{B\}$ : the probability of event $B$;

a.s.: almost surely, or with probability 1;

$I_{B}$ : the indicator function of a set $B$; that is, $I_{B}(x)=1$

if $x \in B$ or otherwise 0 ;

$a \wedge b$ : the minimum of $a$ and $b$.

\section{Definitions of Stability}

We will investigate various types of stabilities in probability for the $n$-dimensional stochastic discrete-time system

$$
x(t+1)=f(x(t), w(t), t), \quad x\left(t_{0}\right)=x_{0},
$$

where $x_{0} \in R^{n}$ is a constant vector. For any given initial value $x\left(t_{0}\right)=x_{0} \in R^{n}$, (6) has a unique solution that is denoted by $x\left(t ; t_{0}, x_{0}\right)$ or $x\left(t ; t_{0}, x_{0}\right)=x(t)$ simply. $w(t)$ is a one-dimensional stochastic process defined on the complete probability space $(\Omega, F, P)$. We assume that $f(0, w(t), t) \equiv 0$ for all $t \in I:=\left\{t_{0}+k: k \in Z^{+}\right\}$, so (6) has the solution $x(t) \equiv$ 0 corresponding to the initial value $x\left(t_{0}\right)=0$. This solution is called the trivial solution or the equilibrium position.

Definition 1. The trivial solution of (6) is said to be stochastically stable or stable in probability if, for every $\varepsilon>0$ and $h>0$, there exists $\delta=\delta\left(\varepsilon, h, t_{0}\right)>0$, such that

$$
P\{|x(t)|<h\} \geq 1-\varepsilon, \quad t \geq t_{0},
$$

when $\left|x_{0}\right|<\delta$. Otherwise, it is said to be stochastically unstable.

If the previous $\delta$ is independent of $t_{0}$, that is, $\delta=\delta(\varepsilon, h)>$ 0 , then the trivial solution of (6) is said to be stochastically uniformly stable in probability.
Definition 2. The trivial solution of (6) is said to be stochastically asymptotically stable in probability if it is stochastically stable, and for every $\varepsilon>0$, there exists $\delta=\delta\left(\varepsilon, t_{0}\right)>0$, such that

$$
P\left\{\lim _{t \rightarrow \infty} x(t)=0\right\} \geq 1-\varepsilon,
$$

when $\left|x_{0}\right|<\delta$.

Definition 3. The trivial solution of (6) is said to be stochastically uniformly asymptotically stable in probability if it is stochastically uniformly stable in probability, and for every $\varepsilon>0, h>0$, there exist $\delta=\delta(\varepsilon, h)>0$ and a $T(\varepsilon)>0$, such that

$$
P\{|x(t)|<h\} \geq 1-\varepsilon, \quad \forall t \geq t_{0}+T(\varepsilon) \text {, when }\left|x_{0}\right|<\delta .
$$

Definition 4. The trivial solution of (6) is said to be stochastically asymptotically stable in the large in probability if it is stochastically stable, and for all $x_{0} \in R^{n}$,

$$
P\left\{\lim _{t \rightarrow \infty} x(t)=0\right\}=1 .
$$

Definition 5. The trivial solution of (6) is said to be uniformly bounded if, for every $\alpha>0$ and $t_{0} \in Z^{+}$, there exists $\beta=$ $\beta(\alpha)>0$, such that

$$
|x(t)|<\beta, \quad \text { a.s. }
$$

when $\left|x_{0}\right|<\alpha$ and $t \geq t_{0}$.

Definition 6. The trivial solution of (6) is said to be stochastically uniformly asymptotically stable in the large in probability if the following are satisfied:

(i) it is stochastically uniformly stable;

(ii) it is uniformly bounded;

(iii) for any $\alpha>0, h>0$, and $\varepsilon>0$, there exists $T(\varepsilon, \alpha)>$ 0 , such that

$$
P\{|x(t)|<h\} \geq 1-\varepsilon, \quad \forall t \geq t_{0}+T(\varepsilon, \alpha),\left|x_{0}\right|<\alpha .
$$

Definition 7. The trivial solution of (6) is said to be $p$ th moment exponentially stable if there exist positive constants $\lambda$ and $C$, such that

$$
E|x(t)|^{p} \leq C\left|x_{0}\right|^{p} e^{-\lambda\left(t-t_{0}\right)}
$$

where $p>0, t \geq t_{0}$, and $x_{0} \in R^{n}$. When $p=2$, it is usually said to be exponentially stable in mean square.

Below, we consider such a continuous function

$$
V(x): R^{n} \longrightarrow R,
$$

with $V(0)=0$, and write

$$
\Delta V(x(t))=V(x(t+1))-V(x(t)) .
$$


Definition 8 (see [13]). A continuous function $\varphi:[0,+\infty) \rightarrow$ $[0,+\infty)$ is said to belong to class $K$ if it is strictly increasing and $\varphi(0)=0$.

Definition 9 (see [13]). A continuous function $V(x)$ defined on $D_{r}$ is said to be positive definite (in the sense of Lyapunov) if $V(0)=0$ and, for some $\varphi \in K$,

$$
V(x) \geq \varphi(|x|) .
$$

A continuous function $V(x)$ defined on $D_{r}$ is said to be negative definite (in the sense of Lyapunov) if $-V(x)$ is positive definite.

Definition 10 (see [13]). A function $V(x)$ defined on $D_{r}$ is said to be radially unbounded if

$$
\lim _{|x| \rightarrow \infty} \inf V(x)=\infty .
$$

Definition 11. A function $V(x)$ defined on $D_{r}$ is said to have infinite small upper bound if there exists $\varphi \in K$ such that

$$
|V(x)| \leq \varphi(|x|) .
$$

\section{Main Results}

In this section, we state our main results in this paper. By using the method of the Lyapunov functionals, some efficient criteria for the stability are obtained.

Theorem 12. If there exists a positive definite function $V(x) \in$ $C^{2}\left(D_{r}\right)$, such that

$$
E[\Delta V(x(t))] \leq 0,
$$

for all $x(t) \in D_{r}$, then the trivial solution of (6) is stochastically stable in probability.

Proof. By the definition of $V(x)$, we obtain that $V(0)=0$ and that there exists a function $\varphi \in K$, such that

$$
V(x) \geq \varphi(|x|), \quad \forall x \in D_{r} .
$$

For any $\varepsilon \in(0,1)$ and $h>0$, without loss of generality, we assume that $h<r$. Because $V(x)$ is continuous, we can find that $\delta=\delta\left(\varepsilon, h, t_{0}\right)>0$, such that

$$
V(x) \leq \varepsilon \varphi(h), \quad \forall x \in D_{\delta} .
$$

It is obvious that $\delta<h$. We fix the initial value $x_{0} \in D_{\delta}$ arbitrarily. Let $\mu$ be the first exit time of $x(t)$ from $D_{h}$; that is,

$$
\mu=\inf \left\{t \geq t_{0}: x(t) \notin D_{h}\right\} .
$$

Let $\tau=\mu \wedge t$, for any $t \geq t_{0}$, we have

$$
\begin{aligned}
V(x(\mu \wedge t))-V\left(x_{0}\right)= & V(x(\tau))-V(x(\tau-1)) \\
& +V(x(\tau-1))-V(x(\tau-2)) \\
& +\cdots+V\left(x\left(t_{0}+1\right)\right)-V\left(x_{0}\right) \\
= & \sum_{t=t_{0}}^{\tau-1} \Delta V(x(t)) .
\end{aligned}
$$

Taking the expectation on both sides, it is easy to see that

$$
E V(x(\mu \wedge t)) \leq V\left(x_{0}\right) .
$$

If $\mu \leq t$ and we note that $|x(\mu \wedge t)|=|x(\mu)|=h$, then

$$
\varphi(h) P\{\mu \leq t\} \leq E\left[I_{\{\mu \leq t\}} V(x(\mu))\right] \leq E V(x(\mu \wedge t)) .
$$

From (21) and (24), we achieve that

$$
P\{\mu \leq t\} \leq \varepsilon .
$$

Letting $t \rightarrow+\infty$, then $P\{\mu<\infty\} \leq \varepsilon$; that is,

$$
P\{|x(t)|<h\} \geq 1-\varepsilon, \quad t \geq t_{0} .
$$

Therefore, the trivial solution of (6) is stochastically stable.

Theorem 13. If there exists a positive definite and infinite small upper bounded function $V(x)$, such that

$$
E[\Delta V(x(t))] \leq 0,
$$

then the trivial solution of (6) is stochastically uniformly stable in probability.

Proof. By the assumptions, there exist $\varphi \in K$ and $\psi \in K$, such that

$$
\varphi(|x|) \leq V(x) \leq \psi(|x|), \quad \forall x \in D_{r} .
$$

Let $\varepsilon \in(0,1)$ and $h>0$ be arbitrary. Without loss of generality, we may assume that $h<r$. We define

$$
\psi(\delta)=\varphi_{h}(\varepsilon) .
$$

Because of $\varphi \in K$, we can obtain $\delta=\psi^{-1}\left(\varphi_{h}(\varepsilon)\right)$, and it has nothing to do with $t_{0}$.

Similar to the proof of Theorem 12, Theorem 13 is established.

Remark 14. We note that $E[\Delta V(x(t))] \leq 0$ in Theorems $12-13$ corresponds to $\mathscr{L} V(x) \leq 0$ in the Itô systems. In Theorem 13, $V(\cdot)$ is not only a positive function, but it is also an infinite small upper bounded function; this is because Theorem 13 is stronger than Theorem 12.

Theorem 15. If there exist a function $\varphi \in K$ and a positive definite function $V(x) \in C^{2}\left(D_{r}\right)$, such that $E[\Delta V(x(t)] \leq$ $-E \varphi(|x(t)|)$ for all $x(t) \in D_{r}$, then the trivial solution of (6) is stochastically asymptotically stable in probability.

Proof. From Theorem 12, we have that the trivial solution of (6) is stochastically stable. Fix $\varepsilon \in(0,1)$ arbitrarily; then there is $\delta_{0}=\delta_{0}\left(\varepsilon, t_{0}\right)>0$, such that

$$
P\left\{|x(t)|<\frac{r}{2}\right\} \geq 1-\frac{\varepsilon}{4},
$$

when $x_{0} \in D_{\delta_{0}}$. 
Fix $x_{0} \in D_{\delta_{0}}$ arbitrarily. By the assumptions on function $V(x)$, we know that $V(0)=0$ and that there exist two functions $\varphi_{1}, \varphi \in K$, such that

$$
\begin{gathered}
\varphi_{1}(|x|) \leq V(x), \\
E[\Delta V(x(t))] \leq-E \varphi(|x(t)|), \quad \forall x \in D_{r} .
\end{gathered}
$$

Let $0<\beta<\left|x_{0}\right|$ arbitrarily, and choose $0<\alpha<\beta, 0<$ $\eta<\alpha$ sufficiently small; because of $V(x)$ being continuous, we can find that $0<\delta=\delta\left(\varepsilon, t_{0}\right)<\delta_{0}$, such that

$$
V(x) \leq \frac{\varepsilon}{4} \varphi(\eta), \quad \forall x \in D_{\delta} .
$$

Define the stopping times

$$
\begin{aligned}
& \mu_{\alpha}=\inf \left\{t \geq t_{0}:|x(t)| \leq \alpha\right\}, \\
& \mu_{r}=\inf \left\{t \geq t_{0}:|x(t)| \geq \frac{r}{2}\right\} .
\end{aligned}
$$

Choose $\theta$ sufficiently large, such that

$$
P\left\{\mu_{\alpha}<\theta\right\} \geq 1-\frac{\varepsilon}{2} .
$$

Let $\tau=\mu_{\alpha} \wedge \mu_{r} \wedge t$, for any $t \geq t_{0}$, we have

$$
\begin{aligned}
0 \leq V(x(\tau))-V\left(x_{0}\right)= & V(x(\tau))-V(x(\tau-1)) \\
& +V(x(\tau-1))-V(x(\tau-2)) \\
& +\cdots+V\left(x\left(t_{0}+1\right)\right)-V\left(x_{0}\right) \\
= & \sum_{t=t_{0}}^{\tau-1} \Delta V(x(t)) .
\end{aligned}
$$

Taking the expectation on both sides, we can derive that

$$
0 \leq E V(x(\tau)) \leq V\left(x_{0}\right)-\varphi(\alpha)\left(\tau-t_{0}\right) .
$$

Hence,

$$
\begin{aligned}
\frac{V\left(x_{0}\right)}{\varphi(\alpha)} & \geq E\left(\mu_{\alpha} \wedge \mu_{r} \wedge t-t_{0}\right) \\
& =E\left(\tau-t_{0}\right) \geq\left(t-t_{0}\right) P\left\{\mu_{\alpha} \wedge \mu_{r} \geq t\right\} .
\end{aligned}
$$

This means that

$$
P\left\{\mu_{\alpha} \wedge \mu_{r}<\infty\right\}=1 .
$$

By (31), $P\left\{\mu_{r}<\infty\right\} \leq \varepsilon / 4$. So

$$
\begin{aligned}
P\left\{\mu_{\alpha}\right. & <\infty\}+\frac{\varepsilon}{4} \geq P\left\{\mu_{\alpha}<\infty\right\}+P\left\{\mu_{r}<\infty\right\} \\
& \leq P\left\{\mu_{\alpha} \wedge \mu_{r}<\infty\right\}=1,
\end{aligned}
$$

which implies that

$$
1-\frac{\varepsilon}{4} \leq P\left\{\mu_{\alpha}<\infty\right\} .
$$

Hence,

$$
\begin{aligned}
P\left\{\mu_{\alpha}<\mu_{r} \wedge \theta\right\} & \geq P\left(\left\{\mu_{\alpha}<\theta\right\} \cap\left\{\mu_{r}=\infty\right\}\right) \\
& \geq P\left\{\mu_{\alpha}<\theta\right\}-P\left\{\mu_{r}<\infty\right\} \geq 1-\frac{3}{4} \varepsilon .
\end{aligned}
$$

Define the two stopping times

$$
\begin{aligned}
\sigma & = \begin{cases}\mu_{\alpha} & \text { if } \mu_{\alpha}<\mu_{r} \wedge \theta, \\
\infty & \text { otherwise },\end{cases} \\
\mu_{\beta} & =\inf \{t>\sigma:|x(t)| \geq \beta\} .
\end{aligned}
$$

Similar to the proof of (24), we can show that, for $t \geq \theta$,

$$
E V(x(\sigma \wedge t)) \geq E V\left(x\left(\mu_{\beta} \wedge t\right)\right) .
$$

If $\mu_{\alpha} \geq \mu_{r} \wedge \theta$, then we note that $\left|x\left(\mu_{\beta} \wedge t\right)\right|=|x(\sigma \wedge t)|=$ $|x(t)|=\eta$, then

$$
E\left[I_{\left\{\mu_{\alpha}<\mu_{r} \wedge \theta\right\}} V\left(x\left(\mu_{\alpha}\right)\right)\right] \geq E\left[I_{\left\{\mu_{\alpha}<\mu_{r} \wedge \theta\right\}} V\left(x\left(\mu_{\beta} \wedge t\right)\right)\right] .
$$

By (31) and $\left\{\mu_{\alpha}<\mu_{r} \wedge \theta\right\} \supset\left\{\mu_{\beta} \leq t\right\}$, we have

$$
\begin{aligned}
\varphi_{1}(\eta) P\left\{\mu_{\beta} \leq t\right\} & \leq E\left[I_{\left\{\mu_{\beta} \wedge t\right\}} V\left(x\left(\mu_{\beta} \wedge t\right)\right)\right] \\
& \leq E V\left(x\left(\mu_{\beta} \wedge t\right)\right) .
\end{aligned}
$$

Together with (33), we get

$$
\frac{\varepsilon}{4} \geq P\left\{\mu_{\beta} \leq t\right\}
$$

Letting $t \rightarrow \infty$, we obtain

$$
\frac{\varepsilon}{4} \geq P\left\{\mu_{\beta}<\infty\right\}
$$

By (42), it follows that

$$
\begin{aligned}
1-\varepsilon & \leq P\left\{\mu_{\alpha}<\mu_{h} \wedge \theta\right\}-P\left\{\mu_{\beta}<\infty\right\} \\
& \leq P\left\{\sigma<\infty, \mu_{\beta}=\infty\right\}
\end{aligned}
$$

This means that

$$
P\left\{\lim _{t \rightarrow \infty} \sup |x(t)| \leq \beta\right\} \geq 1-\varepsilon .
$$

Since $\beta$ is arbitrary, then we have

$$
P\left\{\lim _{t \rightarrow+\infty} x(t)=0\right\} \geq 1-\varepsilon
$$

Theorem 16. If there exist a function $\varphi \in K$ and a positive definite, infinite small upper bounded function $V(x)$, such that $E\left[\Delta V(x(t)] \leq-E \varphi(|x(t)|)\right.$, for all $x(t) \in D_{r}$, then the trivial solution of (6) is stochastically uniformly asymptotically stable in probability. 
Proof. By the assumptions, there exist $\varphi_{1}, \varphi_{2}$, and $\varphi \in K$, such that

$$
\begin{gathered}
\varphi_{1}(|x|) \leq V(x) \leq \varphi_{2}(|x|), \\
E[\Delta V(x(t))] \leq-E \varphi(|x(t)|) .
\end{gathered}
$$

From Theorem 13, we know that the trivial solution of (6) is stochastically uniformly stable. Therefore, for every $\varepsilon>0$ and $h>0$, there exists $\delta=\delta(\varepsilon, h)>0$, such that

$$
P\{|x(t)|<h\} \geq 1-\varepsilon, \quad \forall t \geq t_{0}, \quad\left|x_{0}\right|<\delta .
$$

According to Definition 3 we only need to show that, for every $\varepsilon>0$ and $h>0$, there exist $\delta=\delta(\varepsilon, h)>0$ and $T(\varepsilon)>0$, such that

$P\{|x(t)|<h\} \geq 1-\varepsilon, \quad \forall t \geq t_{0}+T(\varepsilon)$, whenever $\left|x_{0}\right|<\delta$.

We use a contradiction argument; take $T(\varepsilon)=\varphi_{2}\left(\delta_{0}\right) /$ $\varphi(\delta(\varepsilon, h))$; suppose, for any $\tau$, that $t_{0} \leq \tau \leq t_{0}+T(\varepsilon)$, such that $|x(\tau)| \geq \delta(\varepsilon, h)$. By $E[\Delta V(x(t)] \leq-\varphi(|x(t)|)$, we can show that

$$
E[\Delta V(x(t))] \leq-\varphi(|x(t)|) \leq-\varphi(\delta(\varepsilon, h)) .
$$

So

$$
\begin{aligned}
\sum_{t=t_{0}}^{\tau-1} E[\Delta V(x(t))] & \leq \sum_{t=t_{0}}^{\tau-1}-\varphi(\delta(\varepsilon, h)) \\
& =-\varphi(\delta(\varepsilon, r))\left(\tau-t_{0}\right) .
\end{aligned}
$$

That is,

$$
E V(x(\tau))-V\left(x_{0}\right) \leq-\varphi(\delta(\varepsilon, h))\left(\tau-t_{0}\right) .
$$

Thus,

$$
\begin{aligned}
E V(x(\tau)) & \leq V\left(x_{0}\right)-\varphi(\delta(\varepsilon, h))\left(\tau-t_{0}\right) \\
& \leq \varphi_{2}\left(\left|x_{0}\right|\right)-\varphi(\delta(\varepsilon, h))\left(\tau-t_{0}\right) \\
& <\varphi_{2}\left(\delta_{0}\right)-\varphi(\delta(\varepsilon, h))\left(\tau-t_{0}\right)
\end{aligned}
$$

whenever $t_{0} \leq \tau \leq t_{0}+T(\varepsilon)$.

Especially, if $\tau=t_{0}+T(\varepsilon)$, it follows that

$$
E V\left(x\left(t_{0}+T(\varepsilon)\right)\right)<\varphi_{2}\left(\delta_{0}\right)-\varphi(\delta(\varepsilon, h)) T(\varepsilon)=0 .
$$

This contradicts the positive definite property of $V(x)$. Then, we can prove that there exists $t_{1} \in\left[t_{0}, t_{0}+T(\varepsilon)\right]$, such that

$$
\left|x\left(t_{1}\right)\right|<\delta(\varepsilon, h) .
$$

According to Definition 3, we have

$$
P\{|x(t)|<h\} \geq 1-\varepsilon, \quad t \geq t_{1} .
$$

Therefore,

$$
P\{|x(t)|<h\} \geq 1-\varepsilon, \quad t \geq t_{0}+T(\varepsilon) .
$$

The proof is complete.
Remark 17. By comparing Theorems 12-15, we know that $E[\Delta V(x(t))] \leq-E \varphi(|x(t)|)$ guarantees the system to be stochastically asymptotically stable. The difference between Theorems 15 and 16 is that $V(x)$ is additionally required to have an infinite small upper bound in Theorem 16, which ensures the trivial solution of (6) to be stochastically uniformly asymptotically stable in probability.

Theorem 18. If there exist a function $\varphi \in K$ and a positive definite radially unbounded function $V(x) \in C^{2}\left(D_{r}\right)$, such that $E\left[\Delta V(x(t)] \leq-E \varphi(|x(t)|)\right.$, for all $x(t) \in D_{r}$, then the trivial solution of (6) is stochastically asymptotically stable in the large.

Proof. By Theorem 12, we know that the trivial solution of (6) is stochastically stable.

Let $\varepsilon \in(0,1)$ be arbitrary, and fix any $x_{0}$. Since $V(x)$ is radially unbounded, then we can choose $r>\left|x_{0}\right|$ sufficiently large, such that

$$
\inf _{|x| \geq r, t \geq t_{0}} V(x) \geq \frac{4 V\left(x_{0}\right)}{\varepsilon} .
$$

Define the stopping time

$$
\mu_{r}=\inf \left\{t \geq t_{0}:|x(t)| \geq r\right\} \text {. }
$$

Similar to the proof of (24), we can obtain that, for any $t \geq t_{0}$,

$$
V\left(x_{0}\right) \geq E V\left(x\left(\mu_{r} \wedge t\right)\right)
$$

From (63), we have

$$
V\left(x_{0}\right) \geq E V\left(x\left(\mu_{r} \wedge t\right)\right) \geq \frac{4 V\left(x_{0}\right)}{\varepsilon} P\left\{\mu_{r} \leq t\right\} .
$$

Together with (65), it yields that

$$
P\left\{\mu_{r} \leq t\right\} \leq \frac{\varepsilon}{4}
$$

Let $t \rightarrow \infty$; we have $P\left\{\mu_{r}<\infty\right\} \leq \varepsilon / 4$. That is to say,

$$
P\{|x(t)| \leq r\} \geq 1-\frac{\varepsilon}{4}, \quad \forall t \geq t_{0} .
$$

In the same way as that of the proof of Theorem 15 , we can show that

$$
P\left\{\lim _{t \rightarrow+\infty} x(t)=0\right\} \geq 1-\varepsilon
$$

This immediately implies that $P\left\{\lim _{t \rightarrow+\infty} x(t)=0\right\}=1$. The proof is complete.

Theorem 19. If there exist a function $\varphi \in K$ and a positive definite, infinite small upper bound and radially unbounded function $V(x)$, such that $E[\Delta V(x(t)] \leq-E \varphi(|x(t)|)$, for all $x(t) \in D_{r}$, then the trivial solution of (6) is stochastically uniformly asymptotically stable in the large in probability. 
Proof. Under the conditions of Theorem 19, there exist $\varphi_{1}, \varphi_{2}$, and $\varphi \in K$, such that

$$
\begin{gathered}
\varphi_{1}(|x|) \leq V(x) \leq \varphi_{2}(|x|), \\
E[\Delta V(x(t))] \leq-E \varphi(|x(t)|) .
\end{gathered}
$$

By Theorem 13, we know that the trivial solution of (6) is stochastically uniformly stable.

In the following, we first verify that the trivial solution of (6) is uniformly bounded. Actually, for any $\alpha>0, t_{0} \geq 0$, due to $\varphi_{1}, \varphi_{2} \in K$, there exists $\beta=\beta(\alpha)$, such that

$$
\varphi_{1}(\beta)=\varphi_{2}(\alpha), \quad \text { that is, } \beta=\varphi_{1}^{-1}\left(\varphi_{2}(\alpha)\right) \text {. }
$$

It is easy to show that

$$
E V(x(t))-V\left(x_{0}\right)=\sum_{t=t_{0}}^{t-1} E[\Delta V(x(t))] \leq 0, \quad t \geq t_{0}
$$

When $\left|x_{0}\right|<\alpha$, we have

$$
\begin{aligned}
\varphi_{1}(|x(t)|) & \leq E V(x(t)) \leq V\left(x_{0}\right) \\
& \leq \varphi_{2}\left(\left|x_{0}\right|\right)<\varphi_{2}(\alpha) \\
& =\varphi_{1}(\beta) .
\end{aligned}
$$

Because of $\varphi_{1}$ being strictly increasing, so $|x(t)|<\beta$, a.s., $t \geq t_{0}$. This implies that the trivial solution of (6) is uniformly bounded.

We further show that, for every $\alpha>0, \varepsilon>0$, and $h>0$, there exists $T(\varepsilon, \alpha)>0$, such that

$$
\begin{array}{r}
P\{|x(t)|<h\} \geq 1-\varepsilon, \\
\forall t \geq t_{0}+T(\varepsilon, \alpha), \text { whenever }\left|x_{0}\right|<\alpha .
\end{array}
$$

As previously stated the trivial solution of (6) is stochastically uniformly stable. Therefore, for every $\varepsilon>0$ and $h>0$, there exists $\delta=\delta(\varepsilon, h)>0$, such that

$$
P\{|x(t)|<h\} \geq 1-\varepsilon, \quad \forall t \geq t_{0}, \quad\left|x_{0}\right|<\delta .
$$

The rest is similar to the proof of Theorem 16 and is thus omitted.

Remark 20. Theorems 18 and 19 are stronger versions of Theorems 15 and 16 , respectively, where $V(x)$ is additionally required to be a radially unbounded function that is used to prove the stability in the large.

In what follows, we will discuss the pth moment exponential stability for (6).

Theorem 21. Suppose that there exist a function $V(x) \in$ $C^{2}\left(D_{r}\right)$ and positive constants $c_{1}, c_{2}$, and $c_{3}$, such that

$$
\begin{gathered}
c_{1}|x|^{p} \leq V(x) \leq c_{2}|x|^{p}, \\
E[\Delta V(x(t))] \leq-c_{3} E V(x(t)) .
\end{gathered}
$$

Then

$$
E|x(t)|^{p} \leq \frac{c_{2}}{c_{1}}\left|x_{0}\right|^{p} e^{-c_{3}\left(t-t_{0}\right)}, \quad t \geq t_{0}, \forall x_{0} \in R^{n} .
$$

That is, the trivial solution of (6) is pth moment exponentially stable.

Proof. Define the stopping time

$$
\mu_{n}=\inf \left\{t \geq t_{0}:|x(t)| \geq n\right\}, \quad n \geq\left|x_{0}\right| .
$$

It is easy to see that $\mu_{n} \rightarrow \infty$ as $n \rightarrow \infty$ almost surely. By $E[\Delta V(x(t))] \leq-c_{3} E V(x(t))$, we can derive that

$$
\begin{aligned}
& E\left[e^{\mathcal{c}_{3}\left(t \wedge \mu_{n}-t_{0}\right)} V\left(x\left(t \wedge \mu_{n}\right)\right)\right]-V\left(x_{0}\right) \\
& =E\left[e^{c_{3}\left(t \wedge \mu_{n}-t_{0}\right)} V\left(x\left(t \wedge \mu_{n}\right)\right)\right. \\
& -e^{\mathcal{C}_{3}\left(t \wedge-1-t_{0}\right)} V\left(x\left(t \wedge \mu_{n}-1\right)\right) \\
& +e^{\mathcal{C}_{3}\left(t \wedge \mu_{n}-1-t_{0}\right)} V\left(x\left(t \wedge \mu_{n}-1\right)\right) \\
& -e^{\mathcal{G}_{3}\left(t \wedge \tau_{n}-2-t_{0}\right)} V\left(x\left(t \wedge \mu_{n}-2\right)\right) \\
& \left.+\cdots+e^{\mathcal{C}_{3}\left(t_{0}+1-t_{0}\right)} V\left(x\left(t_{0}+1\right)\right)\right]-V\left(x_{0}\right) \\
& =E\left[e^{c_{3}\left(t \wedge \mu_{n}-t_{0}\right)} V\left(x\left(t \wedge \mu_{n}\right)\right)\right. \\
& -V\left(x\left(t \wedge \mu_{n}-1\right)\right) \\
& +e^{\mathcal{C}_{3}\left(t \wedge \mu_{n}-t_{0}\right)} V\left(x\left(t \wedge \mu_{n}-1\right)\right) \\
& -e^{\mathcal{C}_{3}\left(t \wedge \mu_{n}-1-t_{0}\right)} V\left(x\left(t \wedge \mu_{n}-1\right)\right) \\
& \left.+\cdots+e^{\mathcal{C}_{3}} V\left(x\left(t_{0}+1\right)\right)\right]-V\left(x_{0}\right) \\
& =e^{\mathcal{C}_{3}\left(t \wedge \mu_{n}-t_{0}\right)} E\left[\Delta V\left(x\left(t \wedge \mu_{n}-1\right)\right)\right] \\
& +e^{\mathcal{G}_{3}\left(t \wedge \mu_{n}-t_{0}\right)} V\left(x\left(t \wedge \mu_{n}-1\right)\right)\left(1-\frac{1}{e^{c_{3}}}\right) \\
& +\cdots+e^{\mathcal{c}_{3}} E\left[\Delta V\left(x\left(t_{0}\right)\right)\right]+e^{\mathcal{C}_{3}} V\left(x_{0}\right)\left(1-\frac{1}{e^{c_{3}}}\right) \\
& \leq e^{\mathcal{c}_{3}\left(t \wedge \mu_{n}-t_{0}\right)}\left(-c_{3}+1-\frac{1}{e^{\mathcal{c}_{3}}}\right) E V\left(x\left(t \wedge \mu_{n}-1\right)\right) \\
& +\cdots+e^{c_{3}}\left(-c_{3}+1-\frac{1}{e^{c_{3}}}\right) V\left(x_{0}\right) \leq 0 .
\end{aligned}
$$

By $c_{1}|x|^{p} \leq V(x) \leq c_{2}|x|^{p}$, we have that

$$
\begin{aligned}
c_{1} e^{c_{3}\left(t \wedge \mu_{n}-t_{0}\right)} E\left|x\left(t \wedge \mu_{n}\right)\right|^{p} \\
\quad \leq E\left[e^{c_{3}\left(t \wedge \mu_{n}-t_{0}\right)} V\left(x\left(t \wedge \mu_{n}\right)\right)\right] \\
\quad \leq V\left(x_{0}\right) \leq c_{2}\left|x_{0}\right|^{p} .
\end{aligned}
$$

Letting $n \rightarrow \infty$, then

$$
c_{1} e^{c_{3}\left(t-t_{0}\right)} E|x(t)|^{p} \leq c_{2}\left|x_{0}\right|^{p},
$$

which implies (77). 
As a corollary, Theorem 21 yields a sufficient criterion for the exponential stability in mean square sense.

Corollary 22. Suppose that there exist a function $V(x) \in$ $C^{2}\left(D_{r}\right)$ and positive constants $c_{1}, c_{2}$, and $c_{3}$, such that

$$
\begin{gathered}
c_{1}|x|^{2} \leq V(x) \leq c_{2}|x|^{2}, \\
E[\Delta V(x(t))] \leq-c_{3} E|x(t)|^{2} .
\end{gathered}
$$

Then the trivial solution of (6) is exponentially stable in mean square.

\section{Illustrative Examples}

In this section, we present three simple examples to illustrate applications of the stability results developed in this paper. We will let $w(t)$ be a one-dimensional stochastic process defined on the complete probability space $(\Omega, F, P)$, such that $E w(t)=$ 0 and $E[w(t) w(s)]=\delta_{s t}$, where $\delta_{s t}$ is the Kronecker delta.

Example 1. Consider the following equation:

$$
x(t+1)=A x(t)+B x(t) w(t), \quad x\left(t_{0}\right)=x_{0},
$$

where $A$ and $B$ are $n \times n$ matrices. Assume that there is a symmetric positive definite matrix $P$, such that

$$
A^{T} P A+B^{T} P B-P \leq 0 .
$$

Now, define the stochastic Lyapunov function $V(x)=x^{T} P x$. It is obvious that

$$
E[\Delta V(x(t))]=E\left[x^{T}(t)\left(A^{T} P A+B^{T} P B-P\right) x(t)\right] \leq 0 .
$$

By Theorem 12, we conclude that the trivial solution $x \equiv 0$ of (83) is stochastically stable in probability.

Example 2. Consider the following stochastic difference equation:

$$
\begin{aligned}
x(t+1) & =A(t) x(t)+B(t) x(t) w(t) \\
& =[A(t)+B(t) w(t)] x(t) \\
& =H(t, w(t)) x(t),
\end{aligned}
$$

where $A(t), B(t)$, and $H(t, w(t))=A(t)+B(t) w(t)=\left(h_{i, j}(t\right.$, $w(t)))$ are all $2 \times 2$ matrix-valued functions defined on $t=$ $t_{0}, t_{0}+1$, and $t_{0}+2, \ldots, x\left(t_{0}\right)=x_{0} \in R^{n}$. Assume that

$$
\max _{i=1,2} E\left\{\sum_{j=1}^{2}\left|h_{i j}(t, w(t))\right|^{2}\right\}<\frac{1}{2}
$$

for all $x(t) \in R^{2}$.
We define the Lyapunov function $V(x)=\max _{i=1,2}\left\{\left|x_{i}\right|^{2}\right\}$. It is positive definite and radially unbounded. Moreover,

$$
\begin{aligned}
E V(x(t+1))= & \max _{i=1,2} E\left\{\left|\sum_{j=1}^{2} h_{i j}(t, w(t)) x_{j}(t)\right|^{2}\right\} \\
\leq & \max _{i=1,2} E\left\{\sum_{j=1}^{2}\left|h_{i j}(t, w(t))\right|^{2} \sum_{j=1}^{2}\left|x_{j}(t)\right|^{2}\right\} \\
\leq & \max _{i=1,2} E\left\{\sum_{j=1}^{2}\left|h_{i j}(t, w(t))\right|^{2}\right\} \\
& \times \max _{j=1,2} E\left\{2\left|x_{j}(t)\right|^{2}\right\} \\
< & \max _{j=1,2} E\left\{\left|x_{j}(t)\right|^{2}\right\}=E V(x(t)) .
\end{aligned}
$$

That is, $E[\Delta V(x(t))]<0$. By Theorem 18, the trivial solution is stochastically asymptotically stable in the large.

Example 3. Consider a one-dimensional linear stochastic difference equation

$$
x(t+1)=a x(t)+b x(t) w(t)
$$

where $a, b$ are all constants, and $a^{2}<b^{2} / 4$. We assume that there exist positive constants $p$ and $c<1$, such that $5 b^{2}<$ $4(1-c) p$.

We define the Lyapunov function $V(x)=p x^{2}$; then

$$
E[\Delta V(x(t))]=\left(\frac{5 b^{2}}{4}-p\right) E x^{2}(t)<-c p E x^{2}(t) .
$$

By Corollary 22, the trivial solution is exponentially stable in mean square.

\section{Conclusions}

This paper has discussed the stability in probability for stochastic discrete-time systems. Using the method of Lyapunov functionals, some efficient criteria for the stability are obtained. Some results of the stability [13] for stochastic differential equations are generalized to stochastic discretetime systems. There are some interesting problems such as the almost sure exponential stability and the stochastic nonlinear $H_{\infty}$ control that merit further study.

\section{Acknowledgments}

This work is supported by NSF of China (Grants nos. 61174078 and 61170054), Specialized Research Fund for the Doctoral Program of Higher Education (Grant no. 20103718110006), the Research Fund for the Taishan Scholar Project of Shandong Province of China, the SDUST Research Fund (Grant no. 2011KYTD105), and the State Key Laboratory of Alternate Electrical Power System with Renewable Energy Sources (Grant no. LAPS13018). 


\section{References}

[1] D. J. N. Limebeer, B. D. O. Anderson, and B. Hendel, "A Nash game approach to mixed $\mathrm{H}_{2} / \mathrm{H}_{\infty}$ control," IEEE Transactions on Automatic Control, vol. 39, no. 1, pp. 69-82, 1994.

[2] D. Hinrichsen and A. J. Pritchard, "Stochastic $H^{\infty}$ ", SIAM Journal on Control and Optimization, vol. 36, no. 5, pp. 15041538, 1998.

[3] W. Zhang and B.-S. Chen, "On stabilizability and exact observability of stochastic systems with their applications," Automatica, vol. 40, no. 1, pp. 87-94, 2004.

[4] B.-S. Chen and W. Zhang, "Stochastic $H_{2} / H_{\infty}$ control with state-dependent noise," IEEE Transactions on Automatic Control, vol. 49, no. 1, pp. 45-57, 2004.

[5] V. Dragan, T. Morozan, and A.-M. Stoica, Mathematical Methods in Robust Control of Discrete-Time Linear Stochastic Systems, Springer, New York, NY, USA, 2010.

[6] L. Arnold, Stochastic Differential Equations: Theory and Applications, Wiley-Interscience, New York, NY, USA, 1972.

[7] A. Friedman, Stochastic Differential Equations and Their Applications, vol. 2, Academic Press, San Diego, Calif, USA, 1976.

[8] R. Z. Has'minskii, Stochastic Stability of Differential Equations, vol. 7 of Monographs and Textbooks on Mechanics of Solids and Fluids: Mechanics and Analysis, Sijthoff \& Noordhoff, Rockville, Md, USA, 1980.

[9] H. J. Kushner, Stochastic Stability and Control, Academic Press, New York, NY, USA, 1967.

[10] V. B. Kolmanovskii and A. Myshkis, Applied Theory of Functional Differential Equations, Kluwer Academic Publishers, Norwell, Mass, USA, 1992.

[11] G. S. Ladde and V. Lakshmikantham, Random Differential Inequalities, vol. 150 of Mathematics in Science and Engineering, Academic Press, New York, NY, USA, 1980.

[12] S. E. A. Mohammed, Stochastic Functional Differential Equations, Longman, New York, NY, USA, 1986.

[13] X. R. Mao, Stochastic Differential Equations and Applications, Horwood, Chichester, UK, 1997.

[14] V. Dragan and T. Morozan, "Mean square exponential stability for some stochastic linear discrete time systems," European Journal of Control, vol. 12, no. 4, pp. 373-399, 2006.

[15] Y. Huang, W. Zhang, and H. Zhang, "Infinite horizon linear quadratic optimal control for discrete-time stochastic systems," Asian Journal of Control, vol. 10, no. 5, pp. 608-615, 2008.

[16] T. Taniguchi, "Stability theorems of stochastic difference equations," Journal of Mathematical Analysis and Applications, vol. 147, no. 1, pp. 81-96, 1990.

[17] B. Paternoster and L. Shaikhet, "About stability of nonlinear stochastic difference equations," Applied Mathematics Letters, vol. 13, no. 5, pp. 27-32, 2000. 


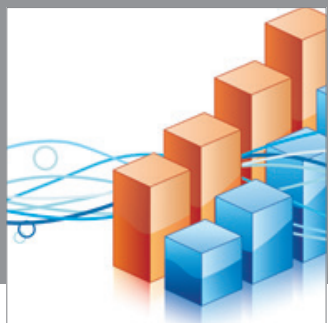

Advances in

Operations Research

mansans

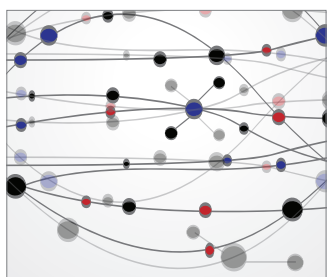

The Scientific World Journal
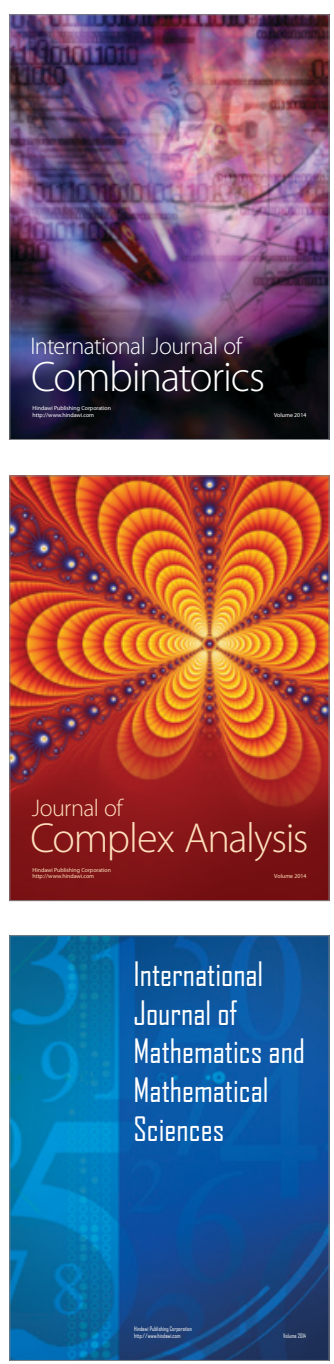
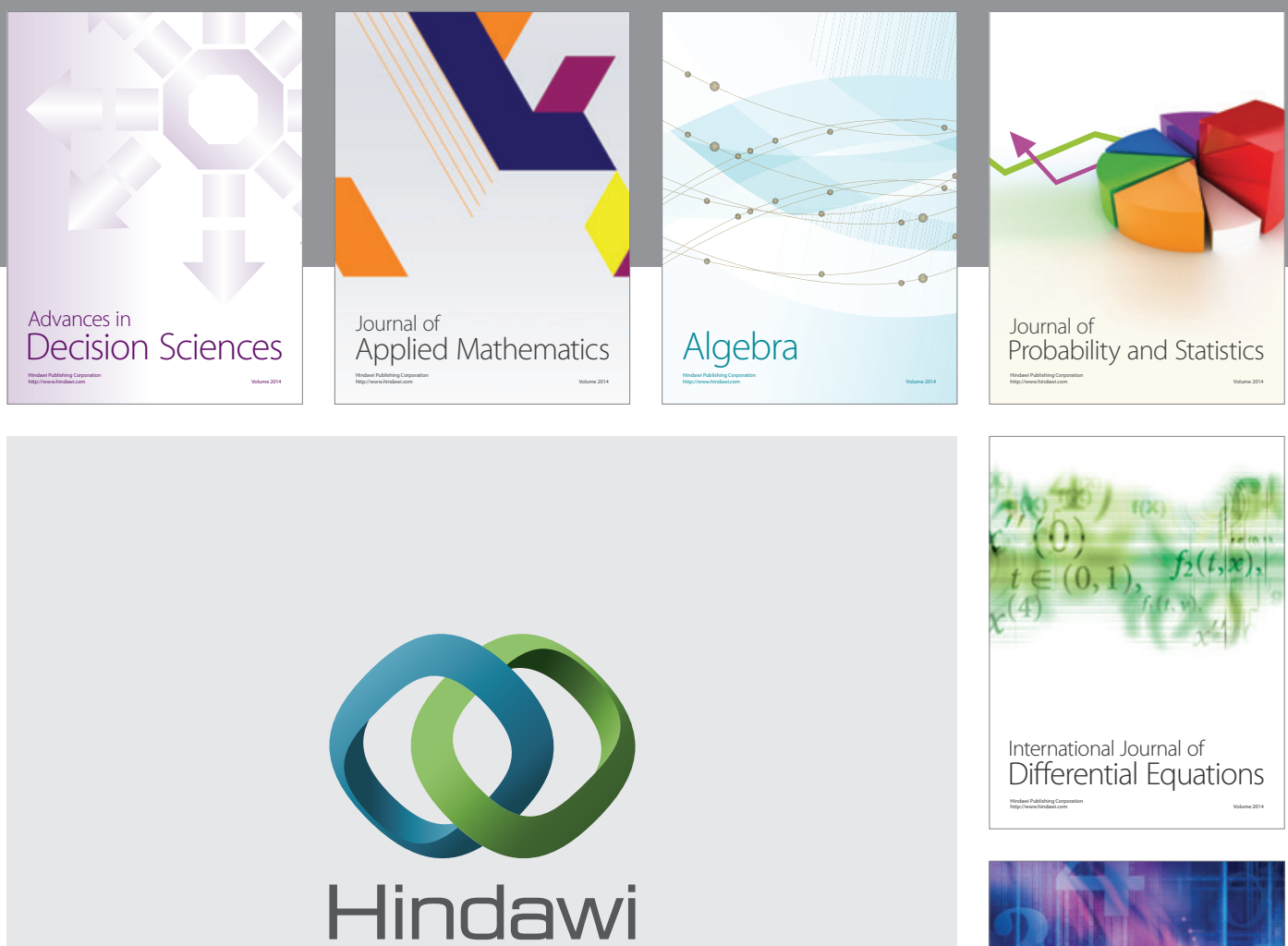

Submit your manuscripts at http://www.hindawi.com
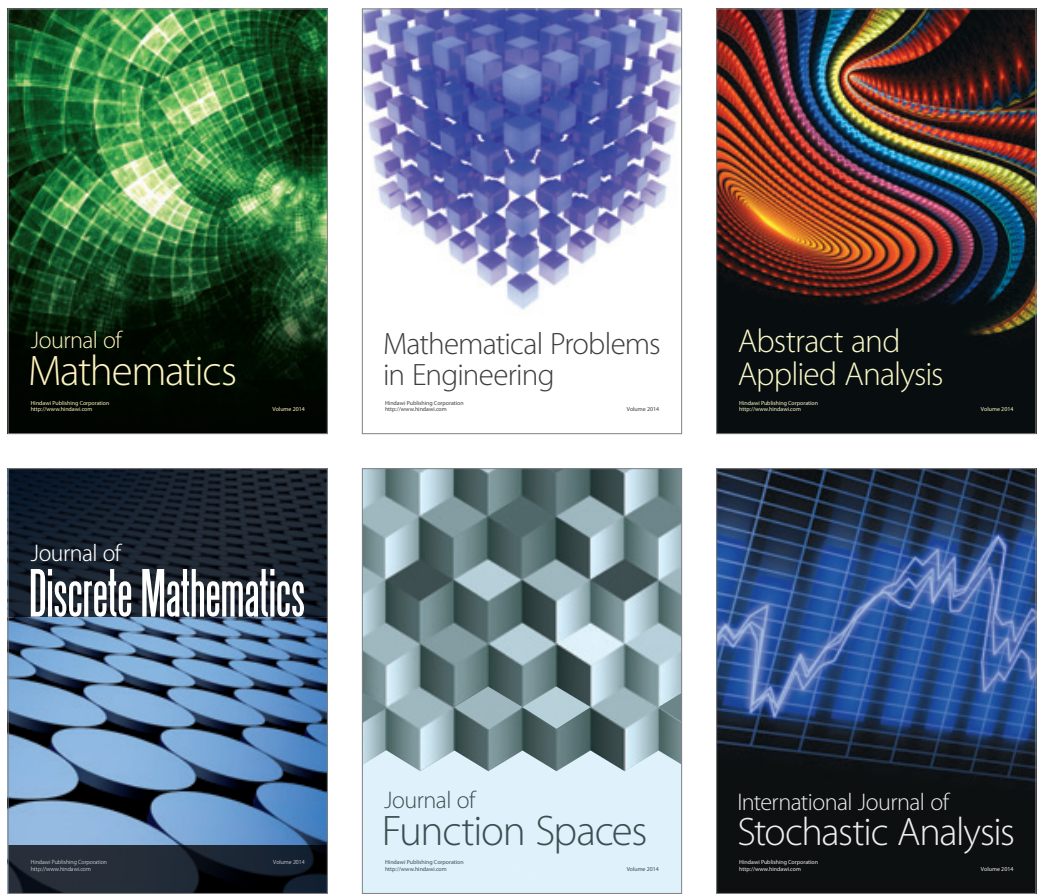

Journal of

Function Spaces

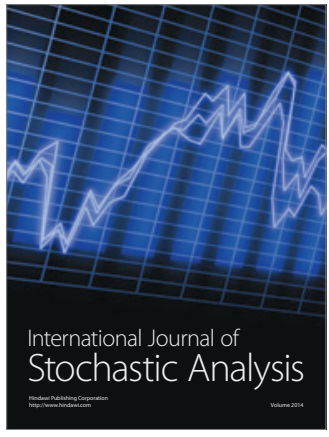

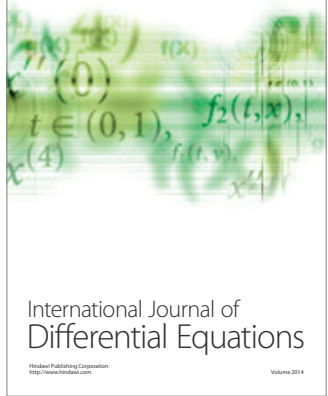
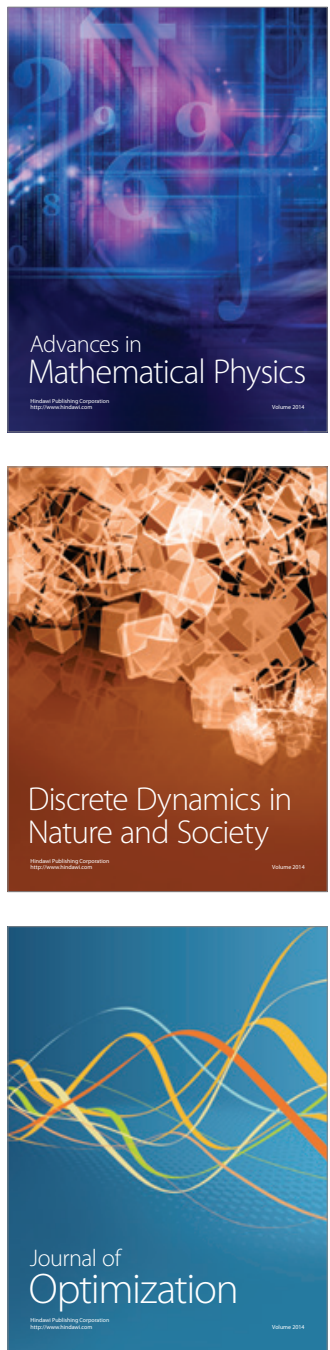